\title{
Frequency of the Cardiovascular Complications during the Chronic Renal Insufficiency with the Service of Nephrology of the National Hospital Donka
}

\author{
Fousseny Diakité1*, Mamadou Saliou Baldé1, Ibrahima Sory Barry², Moussa Traoré1, \\ Alpha Boubacar Bah1', Fenela Mipimbou', Mohamed Lamine Kaba1, Alpha Oumar Bah1 \\ ${ }^{1}$ Unit of Nephrology-Hemodialysis of the National Hospital, Donka, Guinea \\ ${ }^{2}$ Unit of Cardiology of the National Hospital, Ignace Deen, Guinea \\ Email: `fdiak70@gmail.com, ms2balde@yahoo.fr, issobarry@yahoo.fr, mousa11traore@gmal.com, bahalpha427@gmail.com, \\ fenela87@gmail.com,kabalamin@yahoo.fr, bahalphaoumar1@gmail.com
}

How to cite this paper: Diakité, F., Baldé, M.S., Barry, I.S., Traoré, M., Bah, A.B., Mipimbou, F., Kaba, M.L. and Bah, A.O. (2020) Frequency of the Cardiovascular Complications during the Chronic Renal Insufficiency with the Service of Nephrology of the National Hospital Donka. World Journal of Cardiovascular Diseases, 10, 425-431.

https://doi.org/10.4236/wjcd.2020.107041

Received: May 19, 2020

Accepted: July 11, 2020

Published: July 14, 2020

Copyright $\odot 2020$ by author(s) and Scientific Research Publishing Inc. This work is licensed under the Creative Commons Attribution International License (CC BY 4.0).

http://creativecommons.org/licenses/by/4.0/ (c) (i) Open Access

\begin{abstract}
Introduction: Cardiovascular disease has become a major concern for the nephrologist as it is the leading cause of morbidity and mortality in patients with chronic kidney disease, and affects all stages of the disease, including the earliest stages of the disease. The goal of this work was to determine the frequency of cardiovascular complications during chronic kidney failure. Patients and methods: This was a six-month, descriptive cross-sectional study from March 01 to August 31, 2018. It covered all patients with chronic kidney disease hospitalized in the ward during the study period. Included were all chronic kidney failure patients with at least one cardiac and/or vascular complication diagnosed either on clinical examination, and/or paraclinical examination (Electrocardiogram or cardiac ultrasound, vessel echodoppler, scan). Results: During the study period, 84 out of 378 patients or $22.22 \%$ had at least one cardiovascular complication. Cardiovascular complications were hypertrophy of the left ventricle with 49/84 (44 at Electrocardiogram and 5 at cardiac echodoppler), valvulopathy with $33.33 \%$, stroke with $50 \%$ of cases, obliterating arterial disease of the lower limbs $25 \%$, hypokinetic dilated cardiomyopathy with $9 / 36$ cases and pericarditis with $2 / 36$. Conclusion: Cardiovascular complications affect both sexes and all ages. They were dominated by enlarged left ventricle, valvulopathy and dilated cardiomyopathy.
\end{abstract}

\section{Keywords}

Cardiovascular Complications, Chronic Kidney Failure, Donka Conakry 


\section{Introduction}

According to the World Health Organization (WHO), cardiovascular disease is the set of disorders affecting the heart and blood vessels [1], which is the leading cause of death in patients with chronic kidney disease (CKD) [2].

The prevalence of cardiovascular disease is 10 to 30 times higher in patients with chronic kidney disease (CKD) than in the general population. This excess is partly related to an increased prevalence of "classic" risk factors such as high blood pressure, diabetes and dyslipidemia [3]. Cardiovascular disease has become a major concern for the nephrologist as it is the leading cause of morbidity and mortality in patients with CKD, and affects all stages of the disease, including the earliest stages of the disease [4].

In the United States of America, a 2016 study of cardiovascular complications in CKD showed that they were dominated by arteriosclerus heart disease and congestive heart failure with a prevalence of $68.8 \%$ [5]. In France, a 2012 study showed that cardiovascular complications account for $26 \%$ of the causes of death of patients in $\mathrm{CKD}$, the most frequently found were heart failure and coronary pathology [6].

In Algeria (Oran), a 2016 study on the prevalence of cardiovascular complications in CKD reported that the prevalence of cardiovascular complications in their series was $73.2 \%$ which are dominated by Arterial Hypertension in $84.30 \%$, Left Ventricular Hypertrophy (LVH) in $77 \%$ of cases, acute coronary syndromes in $11 \%$ of cases [7]. The frequency and type of cardiovascular complications in CKD patients varied from study to study and from country to country, and we considered appropriately based on our means of exploration, conducting this study. The goal of this work was to determine the frequency of cardiovascular complications during chronic kidney failure.

\section{Patients and Methods}

This was a six-month, descriptive cross-sectional study from March 1 to August 31, 2018. It was performed in the nephrology department of Donka National Hospital and covered all patients with chronic kidney disease hospitalized in the ward during the study period. Included were all chronic kidney failure patients with at least one cardiac and/or vascular complication diagnosed either on clinical examination, and/or paraclinical examination (Electrocardiogram or cardiac ultrasound, vessel echodoppler, CT scan).

Our variables were quantitative and qualitative.

The Electrocardiogram (ECG): looking for:

Left atrial hypertrophy (LAH): retained in front of a too wide $\mathrm{P}$ wave (greater than $120 \mathrm{~ms}$ in DII).

Right atrial hypertrophy (RAH): in front of a too large $\mathrm{P}$ wave (greater than $2.5 \mathrm{mV}$ in DII).

Left ventricular hypertrophy (LVH): increased Sokolow index $=$ SV1 + RV5 or V6 $>35 \mathrm{~mm}$ 
Pericarditis: clinically restrained before pericardial friction and with the ECG we used four stages which were described by Holtzman:

- Stage I: over-shift of the ST segment with a normal or flattened T wave.

- Stage II: the ST segment and the T wave gradually become isoelectric (24th to 48th hour).

- Stage III: the ST segment is isoelectric and under shift of the T wave (for several weeks).

- Stage IV: return to normal of the T wave.

- The left branch block was retained in front of:

- enlargement of the QRS complex.

- exclusive $\mathrm{R}$ or $\mathrm{M}$ aspect in V6 and DI.

- negative T waves in V4, V5, V6.

- The right branch block was retained in front of:

- QRS wide $>0.12 \mathrm{~s}$.

"trailing" waves in DI and V6.

- Atrial Fibrillation was retained before:

- absence of sinus P wave.

- Irregular tachycardia with fine QRS.

- totally irregular R-R intervals.

- fast QRS complexes (100 - 150/min).

Heart rhythm disturbances before:

- the different space between 2 QRS.

- the absence of a P wave before each QRS.

- Tachycardia: it was defined by a heart rate above 100 beats per minute.

- Bradycardia: it was defined by a heart rate below 50 beats per minute.

- The clinical signs of Right Heart Failure and Left Heart Failure were investigated clinically.

- Cardiac ultrasound: looking for a dilation of the heart chambers, an enlarged left ventricle, dilated cardiomyopathy, an ejection fraction ( $\mathrm{EF}<60 \%$ ), a systolic dysfunction of the DV using the TAPSE $<12 \mathrm{~mm}$, a relaxation disorder by calculating the ratio between the speed of the $\mathrm{E}$ wave and that of the $\mathrm{A}$ wave $(\mathrm{E} / \mathrm{A}>2)$.

- Doppler ultrasound of the lower limbs: looking for obliterating arterial disease of the lower limbs, deep vein thrombosis and clinically the sign of positive Homans (pain caused in the calf when the dorsiflexion of the foot).

- Cerebral computed tomography (CT): looking for signs of ischemic or hemorrhagic stroke and in the clinic paralysis of a limb or side, deviation of the mouth, facial paralysis, aphasia, loss of sensitivity.

Data collection and analysis: Data analysis and processing was carried out by the EPI info software in its 7.2 version and the software from the 2013 pack office (Word, Excel) used for document entry, table design.

Ethics; Before undertaking any field activity, the research protocol has been submitted to the nephrology-Hematology-Geriatrics Chair of the Faculty of Health Science and Technology of Gamal Abdel Nasser University in Conakry. 
In the field, written informed consent was also obtained from patients included in our study. Participation in the study was entirely voluntary. No patients who declined to participate were included. Each patient signed or affixed their fingerprint to a voluntary and informed consent card that was read to them by the candidate. For patients unable to read the French language, a translation of the contents of the informed consent form was made in the local language in the presence of a witness or family member.

\section{Results}

In our study, more than $94 \%$ of the patients had a creatinine clearance of 30 $\mathrm{ml} / \mathrm{min}$ or less. The average was $9.19 \mathrm{ml} / \mathrm{min} \pm: 14.15$ (Table 1 ).

Table 2 shows that all patients benefited from the ECG and that LVH was the main complication observed. Fifty percent of the CT patients had cerebrovascular abnormalities.

It emerges from Table 3 that 3 out of 8 patients had vascular anomalies, i.e. $37.5 \%$.

Table 1. Distribution of patients according to general characteristic.

\begin{tabular}{ccc}
\hline SETTINGS & NUMBER & PERCENTYL \\
\hline ECG & 84 & 100 \\
Tachycardia & 29 & 38.16 \\
Left Atrial Enlargement & 3 & 4.35 \\
Left Ventricular Enlargement & 44 & 59.46 \\
Right Ventricular Enlargement & 1 & 1.47 \\
Branch block & 5 & 7.35 \\
Péricarditis & 2 & 2.90 \\
Brain Scanner & 8 & 100 \\
Hemorrhagic stroke & 1 & 12.5 \\
Ischémic stroke & 3 & 37.5 \\
No anomalie & 4 & 50 \\
\hline
\end{tabular}

Table 2. Distribution of patients according to electrocardiogram (ECG) results and CT scan.

\begin{tabular}{|c|c|c|}
\hline Characteristics & Number & Percentyl \\
\hline Frequency & 378 & 100 \\
\hline CKD + Other pathologies without CVC & 294 & 77.78 \\
\hline CKD without CVC & 84 & 22.22 \\
\hline Age & & \\
\hline Medium & \multicolumn{2}{|c|}{$47 \pm 15.27$ ans } \\
\hline Extremes & \multicolumn{2}{|c|}{$15-88$ ans } \\
\hline Sex & 84 & 100 \\
\hline Male & 45 & 53.57 \\
\hline Feminine & 39 & 46.43 \\
\hline
\end{tabular}


Table 3. Distribution of patients according to ultrasound results.

\begin{tabular}{ccc}
\hline Settings & Number & Percentyl \\
\hline Echocardiography & 36 & 100 \\
Hypokinetic dilated heart disease & 9 & 25 \\
Mitral insufficiency & 4 & 11.11 \\
Tricuspid insufficiency & 7 & 19.44 \\
Aortic insufficiency & 1 & 2.77 \\
Left Ventricle Enlargement & 5 & 13.88 \\
Renal Artery Stenosis & 1 & 2.77 \\
Dilatation of the left atrium & 9 & 2.77 \\
Lower limb ultrasound & $\mathbf{8}$ & 100 \\
Stenosis & 1 & 12.5 \\
Obliterating arteriopathy of the lower limbs & 2 & 25 \\
No anomalies & 5 & 62.5 \\
\hline
\end{tabular}

\section{Discussion}

During the study period, 378 patients were hospitalized for all nephrological conditions combined. Of these, 84 in CKD had at least one cardiovascular complication or $22.22 \%$ (Table 1 ). This result is significantly lower than that found by both $\mathrm{AO}$ et al in 2006 [8] 72.95\% of cardiovascular complications. This difference could be explained by the difference in methodology used, including the length of study, sample size and exploration methods used.

The average age of our patients was $47-15.27$ years with extremes of $15-88$ years (Table 1). Our results are lower than those of B. Jerbi et al. in Tunisia [9] which reported, in an analytical retrospective study of the occurrence of major cardiovascular events in CKD patients, an average age of 52.98 years. In the literature, it is reported that the prevalence of CKD increases with age and CKD is a non-modifiable conventional cardiovascular risk factor [10].

Depending on gender we noted a male predominance of $53.57 \%$ versus $46.43 \%$ female with a sex ratio of 1.15 (Table 1). This high prevalence of men could be explained by the fact that most men in our series had a significant cardiovascular risk factor in addition to Arterial Hypertension. Our result is contrary to that of Bah AO, which found in its study $51.69 \%$ of women [8].

This finding could be explained by the fact that the majority of our patients were not sufficiently informed and educated about knowledge of chronic kidney disease as well as cardiovascular risk factors. The clearance of the minimum creatininemia of our patients was $0.9 \mathrm{ml} / \mathrm{min}$ and the maximum was $60 \mathrm{ml} / \mathrm{min}$ with an average of $9.19 \mathrm{ml} / \mathrm{min}$. Clearance of creatininemia of $5 \mathrm{ml} / \mathrm{min}$ was observed in $59.74 \%$ of cases.

Our results are different from those of Bah $\mathrm{AO}$ et al. [8] who reported that $83.3 \%$ of patients had a clearance of creatinine $30 \mathrm{ml} / \mathrm{min}$. Compared to the pa- 
raclinical examinations performed, all patients benefited from ECG, 36 did the morphological examination and 8 did the brain scan. Cardiovascular complications were the hypertrophy of the LAH with 49/84 (44 at ECG and 5 at cardiac echodoppler), valvulopathies with 12 out of 36 who did the cardiac echodoppler, strokes with $4 / 8$ cas, obliterating arterial disease of the lower limbs with $2 / 8$ cases, dilated cardiomyopathy with $9 / 36$ cases and pericarditis with $2 / 36$ (Table 2).

The prevalence and types of cardiovascular complications vary from study to study and depending on the means of exploration used. So W. Berrachdi, N.F. Benatta et al. in 2016 [7] reported 73.2\% of cardiovascular complications and were dominated by arterial hypertension in $84.30 \%$, LAH $77 \%$ of cases, acute coronary syndromes in $11 \%$ of cases, strokes in $17.5 \%$ of cases, obliterating arterial disease of the lower limbs in $13.5 \%$ of cases.

A pathological tracing of ECG was observed in $88.10 \%$ of cases. The main abnormalities were left ventricular hypertrophy (59.46\%), tachycardia (38.16\%) and the branch block (7.35\%) (Table 2). The prevalence of ECG abnormalities in our series was broadly comparable to that found by Chijioke et al. [11] or (86\%) which found a prevalence of left ventricular hypertrophy twice as low as ours or $27.6 \%$. This could be explained by the presence of anaemia and high blood pressure in our patients as the main contributing factors to electrical abnormalities in CKD patients.

However, the ECG's interest in the CKD balance sheet is not limited to the search for a LAH. It also eliminates other abnormalities (conduction, excitability, rhythm disorders, etc.) that are often sub-clinical, making it essential for the therapeutic strategy and follow-up of the CKD patient.

Valvulopathies including mitral, aortic and tricuspid diseases accounted for 49.99\%, dilated cardiomyopathy and LAH were the most dominant in our patients (Table 3). These main morphological abnormalities are also those found in the literature at varying frequencies. In Morocco Ezziani $\mathrm{M}$ et al. [12] found valvulopathies (80\%), LAH (56\%) and dilated cardiomyopathy (12\%).

Study difficulties: The absence of certain examinations such as coronary artery and angioscanne (technical bias) and the high cost of available examinations that did not allow all patients to do them (selection bias) were our difficulties. However, the study identified the prevalence of complications.

\section{Conclusion}

Cardiovascular complications are common during chronic kidney failure. They were dominated by enlarged left ventricle, valvulopathies and dilated cardiomyopathy. A much more in-depth study would help to better diagnose and improve the management of cardiovascular complications.

\section{Conflicts of Interest}

The authors declare no conflicts of interest regarding the publication of this paper. 


\section{References}

[1] WHO about Cardiovascular Disease 2018. https://www.futura-sciences.com

[2] Moustapha, C.M., Tall, L.A., Maria, F., Khodia, F., Moustapha, F., El Hadji Fary, K., Abdou, N. and Boucar, D. (2016) Evaluation of Cardiac Complications among Chronic Hemodialysis in Dakar. Pan African Medical Journal, 23, 43.

[3] Modore, F. (2004) Vascular Risk Factor and Renal Failure. Medicine Science A, 20, 1100-1111.

[4] Go, A.S., Chertow, G.M., Fan, D., McCulloch, C.E. and Hsu, C.Y. (2004) Chronic Kidney Disease and the Risks of Death Cardiovascular Events, and Hospitalization. The New England Journal of Medicine, 351, 1296-305. https://doi.org/10.1056/NEJMoa041031

[5] Cozzolino, M., Galassi, A., Pivari, F., Ciceri, P. and Conté, F. (2017) The Cardiovascular Burden in End-Stage Renal Disease. In: Ronco, C., Ed., Extended Hemodialysis-An Innovative Clinical Approach to Dialysis, Contributions to Nephrology Book 191, Karger, Bale, 44-57. https://doi.org/10.1159/000479250

[6] (2012) Rein (Epidemiology and Information Network in Nephrology). French Registry of Treatments for Chronic Kidney Failure. Annual Report. http://www.agence-biomedecine.fr/IMG/pdf/rapport Rein $2012 \mathrm{~V}$ def.pdf

[7] Ezziani, M., Najdi, A., Mikou, S., Elhassani, A., Akrichi, M.A., Hanin, H., et al. (2014) Echocardiographic Abnormalities in Chronic Hemodialysis Patients: Prevalence and Risk Factors. The Pan African Medical Journal, 18, 216. https://doi.org/10.11604/pamj.2014.18.216.4438

[8] Bah, A.O., Kaba, M.L. and Tossou, E. (2006) Cardiovascular Complications of Chronic Renal Failure in the Nephrology Department of the Donka Teaching Hospital in Conakry. Rev Int Sc Méd, 8, 7-9.

[9] Jerbi, B., Sahnoun, M., Abid, S., Krichene, S., Abid, D., Mallek, S., et al. (2011) Chronic Renal Failure and Myocardial Infarction: Epidemiological, Clinical, Therapeutic and Prognostic Features (about 231 Cases). Annales de Cardiologie et d Angéiologie, 60, 127-134. https://doi.org/10.1016/j.ancard.2010.12.013

[10] Covic, J., Gusbeth-Tatomir, P. and Goldsmith, D.J. (2003) The Challenge of Cardiovascular Risk Factors in End-Stage Renal Disease. Journal of Nephrology, 16, 476-486.

[11] Berrachdi, W. and Benatta, N.F. (2017) Prevalence of Cardiovascular Complications in Chronic Renal Failure on Dialysis in Oran. 13, 389-390.

[12] Chijioke, A.M., Makusidi, P. and Kolo, M. (2012) Electrocardiographic Abnormalities among Dialysis Naive Chronic Kidney Disease Patients in Lorin Nigeria. Annals of African Medicine, 11, 21-26. https://doi.org/10.4103/1596-3519.91011 